\title{
Facilitare la transizione da cattive abitudini a sane abitudini
}

\section{David Mariani}

Healthy Habits Academy Director, Montecatini Terme (PT) - Italy

\begin{abstract}
Facilitating transition from bad to healthy habits
One of the main challenges in the near future, will be to facilitate a wide-spread and generalized change of habits in the population. Chronic diseases risk sending health systems into default. Methods used until today have not worked but recent studies of social psychology and neuroscience show that it is possible to challenge bad habits through new approaches based on the "Nudge Theory". These new approaches are based on principles opposite to those applied up today. They are in fact devoid of prescriptions or impositions but are based on selfempowerment and self-determination of the path towards change. The Healthy Habits Approach is an interesting experiment of active social change in Italy.
\end{abstract}

Keywords: Healthy habits, Nudge Theory, Social change, Transition

Un cambiamento generalizzato di abitudini nella popolazione diventa una delle sfide più urgenti del prossimo futuro.

La pandemia di Covid-19 ci ha messo di fronte, amplificandolo al massimo, al problema di quanto siano importanti le condizioni di salute generale della popolazione (1).

Lo stile di vita moderno fatto di sedentarietà, cattive abitudini alimentari, inquinamento ambientale e stress ricorrenti ha portato, in meno di 50 anni, a un enorme incremento di patologie croniche (2).

Il problema non colpisce solo la popolazione anziana, di per sé più esposta per condizioni biologiche svantaggiose, ma anche le nuove generazioni.

L'infiammazione cronica è sempre più diffusa nella popolazione, indipendentemente dall'età, e trae origine proprio dai cattivi stili di vita, preparando il terreno a qualunque patologia (3).

Se non invertiamo la rotta delle abitudini in tempi rapidi andremo a sbattere contro un problema sociosanitario enorme.

Received: February 22, 2021

Accepted: February 25, 2021

Published online: March 10, 2021

Indirizzo per la corrispondenza:

David Mariani

Healthy Habits Academy

Via Bacci 21

51016 Montecatini Terme (PT) - Italy

d.mariani@healthyhabits.it
La fotografia che ci fornisce l'Istituto Superiore di Sanitá è preoccupante: tre bambini su quattro non raggiungono $i$ minimi di attività fisica consigliata dall'OMS e quasi un terzo dei bambini è in sovrappeso. L'incremento di una patologia come il diabete mellito di tipo 2 è stato del 415\%, da cento milioni di malati a quattrocentoquindici milioni in soli 35 anni (4).

II $40 \%$ della popolazione italiana soffre di patologie croniche e il $30 \%$ ha almeno due patologie (5). Oggi sappiamo quanto abitudini e stili di vita siano decisivi nella prevenzione delle stesse e nel contenimento delle comorbilità.

Le strategie sino a oggi adottate per cercare di convincere le persone a cambiare stile di vita non hanno dato i risultati sperati.

Chiedere alle persone di cambiare usando prescrizioni e coercizioni non funziona per diversi motivi, che traggono le loro origini dalla psicologia e dalla neurobiologia $(6,7)$.

Negli ultimi anni, la ricerca ha fatto importanti acquisizioni in questa direzione grazie agli studi di psicologia sociale economia comportamentale e neuroscienze (8). Viceversa, ci sono evidenze che indurre un cambiamento generalizzato di abitudini potrebbe essere più semplice di quanto crediamo, se vengono utilizzate strategie diverse e complementari tra loro.

I premi Nobel 2017, Richard Thaler e Cass Sustein, hanno dimostrato, con la Nudge Theory, che sottoporre le persone a continue "spintarelle gentili" usando sistemi semplici di induzione al cambiamento può funzionare sui grandi numeri.

Test eseguiti nelle mense e nei distributori automatici, per esempio, hanno chiarito che l'essere umano è abitudinario e 
compie spesso scelte inconsapevoli. La semplice sostituzione degli snack dolci con delle mele verdi nello stesso scaffale ha portato moltissime persone ad acquistarle. Stessa cosa è accaduta nei test eseguiti nelle mense; è bastata la semplice sostituzione di una parte dei cibi ad alta densità calorica con frutta verdure e ortaggi per raddoppiare il consumo di frutta e verdura.

Questi test ci dicono che i contesti possono giocare un ruolo importante nelle scelte individuali, indirizzandole, in parte.

In Italia, dal 2014 è attivo un progetto sociale (Healthy Habits), che mira a facilitare la transizione della popolazione verso sane abitudini (8-14).

Partendo proprio dalle debolezze mostrate dai sistemi prescrittivi e impositivi, è stato sviluppato un approccio metodologico al cambiamento che sfrutta principi opposti, come aggiungere anziché levare, nessuna imposizione né privazione, ma tante spintarelle gentili e una totale autodeterminazione del percorso.

Per facilitare il cambiamento, $\mathrm{HH}$ utilizza un percorso interdisciplinare che, per motivi evolutivi, è già presente dentro l'essere umano, sfruttando i quattro ambiti principali in grado di incidere sulla salute umana: ambiente, fisiologia, nutrizione e relazioni sociali (15-19).

Quattro soluzioni diverse a un unico problema, in grado di influenzarsi l'una con l'altra grazie all'interazione che l'uomo e la sua fisiologia hanno con questi driver evolutivi, come dimostrato da numerose recenti ricerche delle principali scuole di medicina mondiali (20-24).

Le chiavi per il successo nel cambiamento dello stile di vita della popolazione vanno in questa direzione, come già verificato e auspicato nelle recenti Linee Guida italiane e internazionali per la promozione dell'attività fisica (25).

Per riuscire a interiorizzare un cambiamento dobbiamo aiutare a esperire situazioni concrete aiutando le persone a riprendere possesso del proprio corpo e far sperimentare le sensazioni positive che possono arrivare dalla pratica di sane abitudini a dosi corrette. Uno dei grandi problemi, infatti, che ha contribuito a fermare milioni di persone è, per esempio, il dosaggio sbagliato dell'esercizio fisico e il voler passare troppo rapidamente da sedentari assoluti ad atleti provetti.

Dosi eccessive di attività fisica rispetto alle condizioni di partenza provocano, infatti, reazioni di rifiuto a causa del dolore e della fatica.

Al contrario, la pratica di attività semplici come il cammino anche per poche decine di minuti permette, se perpetrata, di garantire sia protezione per la salute che una piacevole sensazione di benessere in grado di aumentare la motivazione a continuare. La riattivazione dalla sedentarietà è il passaggio chiave, perché in grado di stimolare, per motivi ancora poco conosciuti, altri comportamenti virtuosi, come un miglioramento delle abitudini alimentari e migliori relazioni sia con le persone che con l'ambiente.
Solo i meccanismi di self-empowerment, uniti a una conoscenza e a una responsabilità sociale accresciute, possono, infatti, mettere in moto un cambiamento generalizzato e quanto mai auspicato di intere popolazioni, per il nostro bene e per quello dell'ecosistema che pazientemente ancora ci ospita.

\section{Disclosures}

Conflict of interest: The authors declare no conflict of interest. Financial support: This research received no specific grant from any funding agency in the public, commercial, or not-for-profit sectors.

\section{Bibliografia}

1. Brawner CA, Ehrman JK, Bole S, et al. Inverse Relationship of Maximal Exercise Capacity to Hospitalization Secondary to Coronavirus Disease 2019. Mayo Clin Proc. 2021;96(1):32-39. CrossRef PubMed

2. Prevenire le malattie croniche: un investimento vitale. Rapporto Globale dell'Organizzazione Mondiale della Sanità. Online

3. Calder PC, Albers R, Antoine JM, et al. Inflammatory disease processes and interactions with nutrition. Br J Nutr. 2009; 101(S1)(suppl 1):S1-S45. CrossRef PubMed

4. World Diabetes Federation. 2017

5. Osservatorio della Salute. 2017.

6. Modelli teorici, competenze e strategie per promuovere il cambiamento a favore di stili di vita salutari. Online

7. Online

8. Thaler RH. The Winner's Curse: Paradoxes and Anomalies of Economic Life. Princeton: Princeton University Press; 1992.

9. Thaler RH. Advances in Behavioral Finance. New York: Russell Sage Foundation; 1993.

10. Thaler RH. Quasi Rational Economics. New York: Russell Sage Foundation; 1994.

11. Thaler RH. (Roundtable Series in Behavioral Economics). Princeton: Princeton University Press; 2005. Advances in Behavioral Finance; vol II.

12. Thaler RH, Sunstein C. (updated edition). Nudge: Improving Decisions About Health, Wealth, and Happiness. New York: Penguin; 2009.

13. Thaler RH. Misbehaving: The Making of Behavioral Economics. New York: W. W. Norton \& Company. 2015;ISBN 978-0-39308094-0 (Ed. italiana: Misbehaving. Torino, Giulio Einaudi Editore. 2018;ISBN 978-88-06-22853-8).

14. Thaler RH, Sunstein C. Nudge - La spinta gentile. Milano, Giacomo Feltrinelli Editore. 2009.

15. Online

16. Mariani D, Capitanini A. Clima ambientale e clima emotivo: due pilastri evolutivi poco tangibili ma tremendamente importanti per la nostra vita. G Clin Nefrol Dial. 2020;32:11-14. CrossRef

17. Capitanini A, Mariani D. Born to run ... ma anche per camminare. Attività motoria, il primo pilastro dell'evoluzione umana. G Tec Nefrol Dial. 2019;31(2):142-145. CrossRef

18. Mariani D, Capitanini A. Il cibo per la vita. G Tec Nefrol Dial. 2019;31(3):207-209. CrossRef

19. Mariani D. La prevenzione come valore sociale. Introduzione alla problematica. G Tec Nefrol Dial. 2019;31(1):72-74. CrossRef

20. Bekalu MA, McCloud RF, Minsky S, Viswanath K. Association of social participation, perception of neighborhood social cohesion, and social media use with happiness: evidence of trade-off (JCOP-20-277). J Community Psychol. 2021;49(2): 432-446. CrossRef PubMed 
21. Denckla CA, Cicchetti D, Kubzansky LD, et al. Psychological resilience: an update on definitions, a critical appraisal, and research recommendations. Eur J Psychotraumatol. 2020;11(1): 1822064. CrossRef PubMed

22. Baden MY, Kino S, Liu X, et al. Changes in plant-based diet quality and health-related quality of life in women. Br J Nutr. 2020;124(9):960-970. CrossRef PubMed

23. Boehm JK, Qureshi F, Chen Y, et al. Optimism and Cardiovascular Health: Longitudinal Findings From the Coronary Artery
Risk Development in Young Adults Study. Psychosom Med. 2020;82(8):774-781. CrossRef PubMed

24. Kubzansky LD, Boehm JK, Allen AR, et al. Optimism and risk of incident hypertension: a target for primordial prevention. Epidemiol Psychiatr Sci. 2020;29:e157. CrossRef PubMed

25. Linee di indirizzo sull'attività fisica per le differenti fasce d'età e con riferimento a situazioni fisiologiche e fisiopatologiche e a sottogruppi specifici di popolazione. Online 